\title{
Brain regions involved in spatial frequency discrimination: evidence from fMRI
}

Received: 27 September 1999 / Accepted: 17 February 2000 / Published online: 18 April 2000

(C) Springer-Verlag 2000

\begin{abstract}
The cortical areas underlying successive spatial-frequency discrimination were explored using functional magnetic resonance imaging (fMRI). In a steadystate, block-design paradigm, 12 subjects viewed a single fixation cross during a rest period, followed by an activation period consisting of the presentation of horizontal (distractors) and vertical (targets) sinewave gratings. Two tasks were performed: in the control task, subjects pressed a button after the second vertical grating was presented within each trial; in the discrimination task, subjects decided which target grating had the higher spatial frequency. Post-processing consisted of off-line image registration to correct for head motion, spatial and temporal smoothing, and cross-correlation between each voxel time course and a phase-shifted stimulus time profile. The results indicate that striate, extrastriate, parietal, and prefrontal areas show significant BOLD (blood oxygen level dependent) effects during both discrimination and control tasks, with consistently higher activity levels in the discrimination task.
\end{abstract}

Key words Functional magnetic resonance imaging (fMRI) - Perceptual discrimination - Spatial frequency · Human $\cdot$ Visual cortex $\cdot$ Psychophysics

\section{Introduction}

Perception is a complex endeavor in which on-line processing of visual signals is matched against visual memory representations in parallel with, and embedded in, discrimination and decision processes, language and categorical processes, and preparation for response execu-

M.W. Greenlee

Cognitive Neuroscience, Department of Psychology,

University of Oldenburg, A6 2-209, 26111 Oldenburg, Germany

S. Magnussen $\cdot$ I. Reinvang

Department of Psychology, University of Oslo, Norway

M.W. Greenlee

Department of Neurology, University of Freiburg, Germany tion. Perception in the natural environment must, thus, activate major portions of the cerebral cortex. To isolate some of the components of the perceptual process and the associated brain areas, recent human brain-mapping studies have used very simple perceptual tasks, such as the discrimination of orientation, spatial frequency, or motion. The rationale behind this approach is that such tasks highlight the essential elements of perceptual discrimination without recruiting higher-order verbal or categorical processes (Reinvang et al. 1998; Orban and Vogels 1998). Experimental and control conditions may be designed that differ with respect to the task requirements, but are based on identical stimulus displays; in addition, the brain mechanisms of the sensory analysis of dimensions such as orientation, spatial frequency, and motion have been studied by psychophysics, neurophysiology, and neuropsychology.

Most studies using this research strategy have been conducted by a single group of researchers (Orban et al. 1997, 1998; Cornette et al. 1998; Dupont et al. 1998), who have used positron emission tomography (PET) and functional magnetic resonance imaging (fMRI) to identify the brain regions activated in orientation and motion discrimination tasks. These studies show a complex pattern of results, with a consistent task-dependent activation related to discrimination in extrastriate areas in the middle fusiform gyrus [Brodmann area (BA) 19/37] and in several prefrontal areas. This pattern of results was confirmed in an fMRI experiment on five subjects, who additionally showed activation of parietal regions in the discrimination task (Cornette et al. 1998). Parietal regions have also been implicated in delayed discrimination tasks by event-related-potential (ERP) experiments. Reinvang et al. (1998) performed a source localization analysis (BESA; Scherg 1996) of ERP responses recorded in a delayed spatial frequency discrimination task with a variable interstimulus interval. They found that a model with four sources localized over the occipital, temporal, and parietal regions provided a good fit to the data for both short and long interstimulus intervals, with the activity profile of the parietal source be- 
ing modulated according to the duration of the interstimulus interval.

In the present experiment, we studied fMRI correlates of successive discrimination of spatial frequency, exploring BOLD (blood oxygen level dependent) responses to a task requiring discrimination of spatial frequency as compared with a task requiring the detection of the mere presence of the same stimuli. Differences in the activation patterns arising in the experimental conditions would, thus, be task dependent rather than stimulus dependent.

\section{Materials and methods}

\section{MR imaging}

Magnetic resonance imaging was performed with a 1.5 Tesla clinical scanner, Siemens Magnetom Vision, equipped with an echoplanar-imaging- (EPI-) booster for fast gradient switching (Siemens, Erlangen) and a full-head radio-frequency (RF) receivetransmit headcoil. High-resolution, sagittal T1-weighted images were acquired with the MP-RAGE (magnetization prepared, rapid acquisition gradient echo) sequence to obtain a three-deminsional anatomical model of the head and brain. We defined the anteriorposterior commissural (AC-PC) plane (Talairach and Tournoux 1988) and report all findings in this coordinate system. Shimming was performed for the entire brain using an auto-shim routine, which yielded satisfactory magnetic-field homogeneity.

Functional imaging was performed with $\mathrm{T} 2 *$-weighted, gradient-recalled EPI. The technical data for the functional measurements were TE $66 \mathrm{~ms}$, TR $3 \mathrm{~s}$, flip angle $90^{\circ}$, field of view $256 \mathrm{~mm}$, matrix $128 \times 128$, resulting in a voxel size of $2 \times 2 \times 4 \mathrm{~mm}$. The stimulation protocol consisted of twelve 30-s intervals with six alternating periods of rest (off) and stimulation (on). This protocol yielded 125 echo-planar volumes acquired over a 6-min period.

To minimize head motion, the subject's head was fixed with a vacuum cap. Despite these precautions, residual head motion was still evident in some of the image data. In-plane motion could be corrected by applying an image-alignment algorithm (Cox 1996). Excessive out-of-plane motion could not be corrected by this algorithm. However, we could detect such head motion, and the images affected were removed from further analysis. The effects of the gradient noises were reduced by having the subject wear either ear plugs or sound-dampening headphones.

BOLD contrast was extracted from the image time series using correlation analysis. Activations with signal changes correlating 0.5 or better with the stimulus time course are highlighted in Fig. 2. Region-of interest (ROI) analyses were performed interactively using BrainTools (Smith et al. 1998). ROIs were defined as voxel clusters $(4 \times 4$ or greater) that were significantly activated (correlation $>0.5$ ) either in the control or the experimental task or both. The averaged position of each ROI for each hemisphere was determined for each subject. Statistical analysis of the activation levels for all ROI were performed off-line on the group data using the program StatView (version 4.5, Abacus Concepts).

\section{Visual stimulation and perceptual tasks}

The subjects viewed the stimuli with a mirror adjusted to allow a maximum field of view. The stimuli were created on a Visual Stimulus Generator graphics card (Cambridge Research) and projected (LCD projector, Panasonic) onto a transluminant screen, which was mounted at the back of the gantry. The image subtended $30 \times 30^{\circ}$ of visual angle $(800 \times 600$ pixels $)$ at a viewing distance of $1.2 \mathrm{~m}$. Reference and test gratings were vertical and, during the experimental run, they were interleaved sequentially with horizontal (distractor) gratings.

The temporal sequence of the stimulus display is shown in Fig. 1. Vertical reference and test gratings were presented sequentially for $150 \mathrm{~ms}$ with an interstimulus interval (ISI) of about $800 \mathrm{~ms}$, and they were interleaved with three horizontal distractors. The relative positions of the distractors and the second vertical (test) grating was randomized over trials. The following sequences were possible: V-V-H-H-H, V-H-V-H-H, V-H-H-V-H, and $\mathrm{V}-\mathrm{H}-\mathrm{H}-\mathrm{H}-\mathrm{V}$, where $\mathrm{V}$ and $\mathrm{H}$ stand for the vertical and horizontal orientation of the gratings. These possible sequences were randomized within each block of trials. Reference and test spatial frequencies always differed by $\pm 10 \%$, the spatial frequency of the gratings was 2 cycles/deg. There were two experimental tasks. In the control task, the subject simply responded to the second presentation of the vertical grating. In the discrimination task, the subject made a two-alternative, forced-choice decision on which of the two vertical gratings, reference or test, had the higher spatial frequency. Performance outside of the scanner corresponded to approximately $90 \%$ correct on the discrimination task. The subject was asked to respond as quickly and accurately as possible by pushing either of two buttons. Accuracy and choice reaction times (RT) were recorded during the fMRI measurements. The experimental session, including preparation for MRI recording, lasted about $1 \mathrm{~h}$. Twelve subjects participated, eight female, four male.

\section{Results}

The performance on the discrimination task in the scanner was similar to that measured outside the scanner (mean performance $=83 \%$ correct), the choice reaction times for the control and discrimination tasks were $751 \pm 16.7 \mathrm{~ms}$ and $929.6 \pm 23.1 \mathrm{~ms}$, respectively $\left(F_{1.58}=26.26, P<0.0001\right)$. Thus, subjects spent, on average, $178 \mathrm{~ms}$ more on the discrimination of spatial frequency than they did on identification of orientation.

An example of the fMRI activation pattern in a single subject is illustrated in Fig. 2. The region-of-interest analyses are summarized in Fig. 3, plotting the mean ROI activation weighted by the standardized correlation coefficient for the perceptual discrimination and control
Fig. 1 Stimulus configuration and the time course of stimulation in a given trial. $R$ Reference, $T$ target, $D$ distractor, Resp. response period. For more details, see text

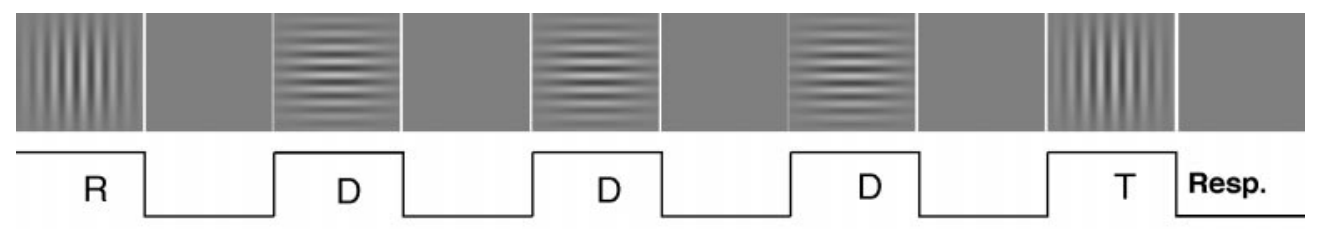



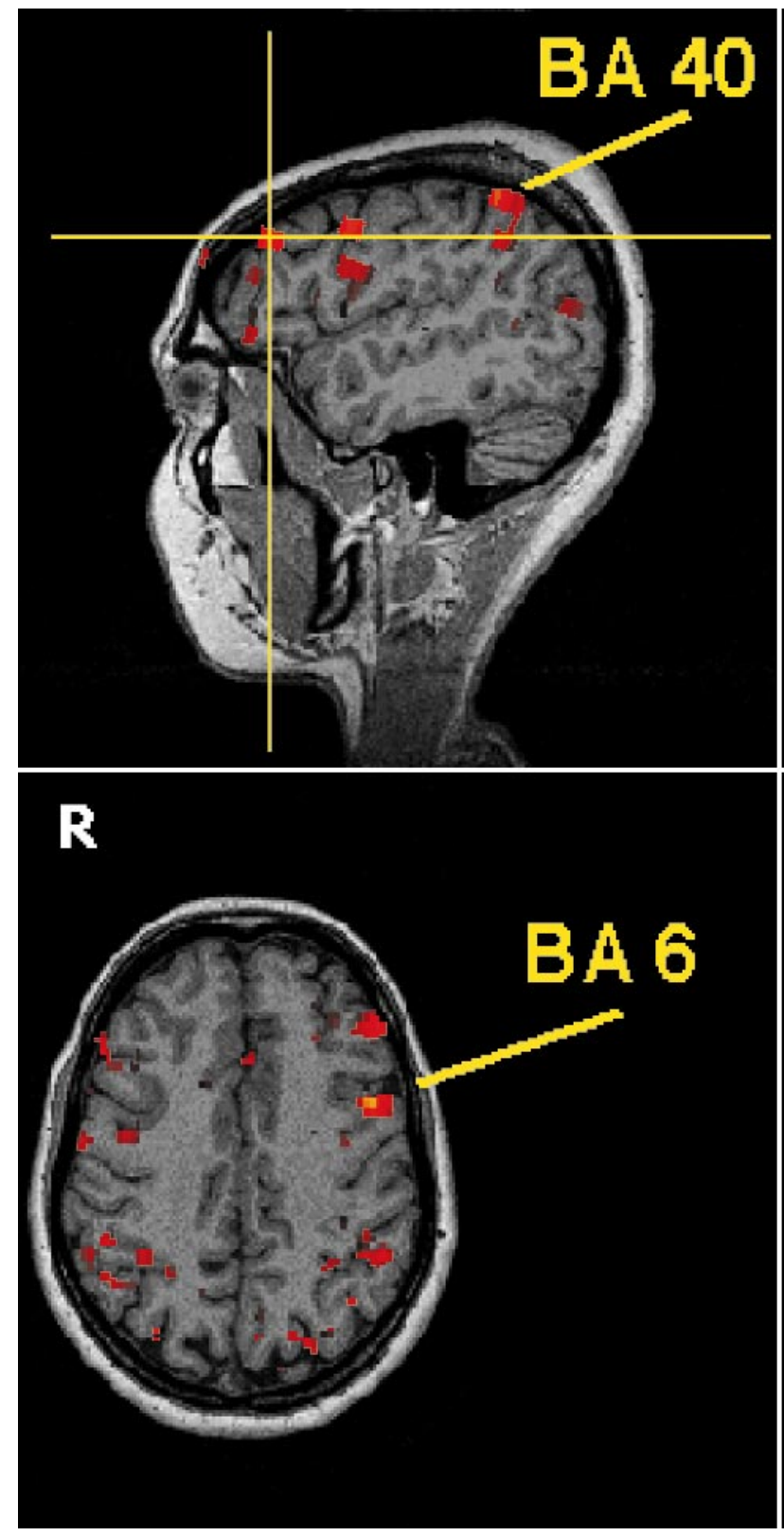

Fig. 2 Example of parietal and prefrontal activations during the spatial-frequency discrimination task. The mean Talairach coordinates of all regions-of-interest are given in Table 1.The top right panel shows a three-dimensional view of one subject and the green lines denote the position of the acquired echo-planar-image (EPI) volume

tasks, with separate graphs for the left and right hemispheres. The corresponding Talairach coordinates are shown in Table 1. Task-dependent activity was observed bilaterally in several regions indicated by previous PET findings, including occipital cortex (BA 17/18) and occipito-temporal regions (BA 19/37), parietal cortex (BA 7/40), and prefrontal (BA 6/9/32) cortex. In all of these regions, BOLD responses were higher for the discrimination task than for the control task $\left(F_{1,7}=12.44\right.$, $P<0.001)$. Activity levels are quite similar in the left and

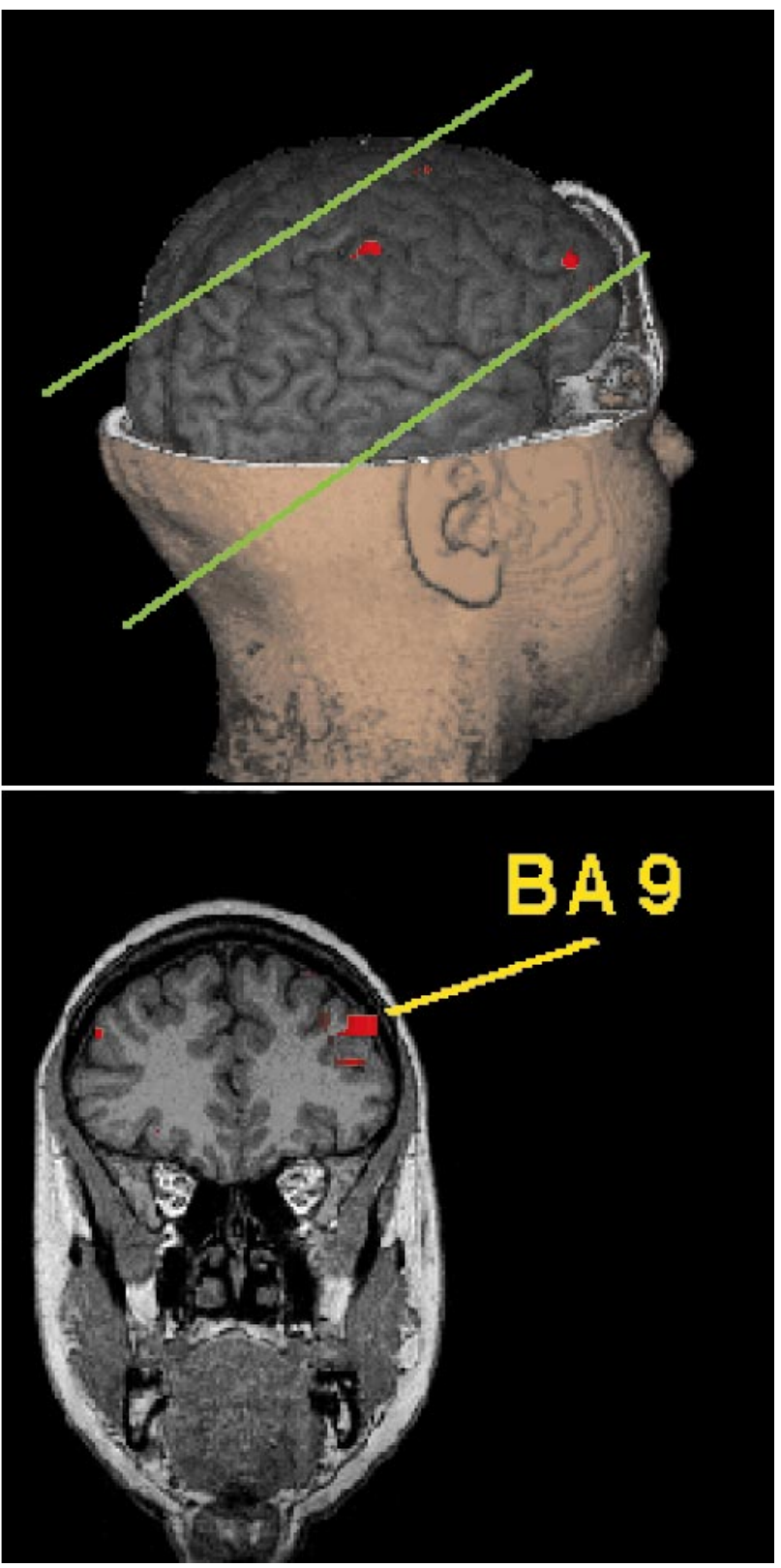

right hemispheres, except for insular cortex, where activity was only observed in the right hemisphere.

\section{Discussion}

The results of fMRI show that areas beyond the occipital cortex are active and involved in perceptual discrimination of spatial frequency, with the striate-extrastriate, parietal, and prefrontal regions showing enhanced activation during perceptual discrimination. In general, our results confirm those of previous brain-imaging experiments on the perceptual discrimination of elementary attributes or dimensions of the visual stimulus (Orban et al. 1997, 1998; Cornette et al. 1998; Dupont et al. 1998). These studies showed that extrastriate occipital areas, especially the middle fusiform gyrus of the right 
Fig. 3a, b Results of the ANOVA [mean regions-ofinterest activation $(R O I)$ averaged over 12 subjects] for the control and memory tasks. a shows the results for the left, b for the right hemisphere. $S F$-discr Spatial frequency discrimination, FEF frontal eye field a)

Left Hemisphere

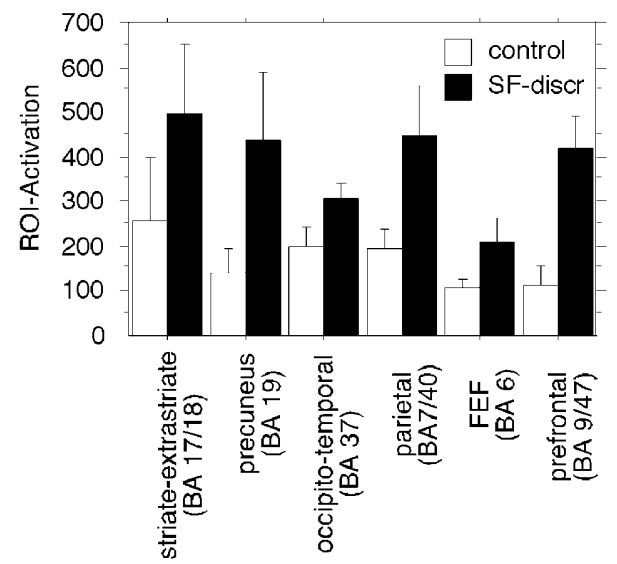

b)

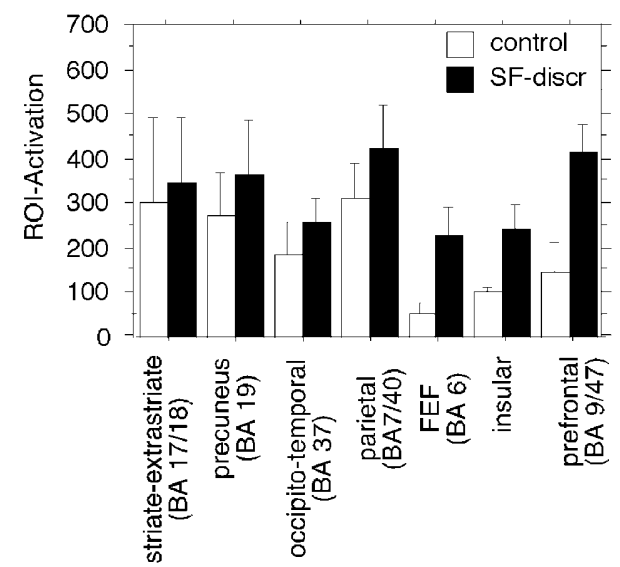

Table 1 Talairach coordinates $(\mathrm{mm}$ deviation from the anterior commissure) for the regions-of-interest, showing significant activation (minimum voxel cluster: $4 \times 4$ ) for the left $(L H)$ and right $(R H)$ hemisphere. The number of subjects that showed a significant effect (comparison between rest and activation in the spatialfrequency discrimination task) is signified by $n$

\begin{tabular}{|c|c|c|c|c|c|c|c|c|}
\hline \multirow{2}{*}{$\begin{array}{l}\text { Brodmann } \\
\text { area }\end{array}$} & \multicolumn{4}{|c|}{ LH } & \multicolumn{4}{|c|}{$\mathrm{RH}$} \\
\hline & $n$ & $\mathrm{x}$ & $\mathrm{y}$ & $\mathrm{z}$ & $n$ & $\mathrm{x}$ & $\mathrm{y}$ & z \\
\hline 6 & 3 & 45 & 3 & 40 & 3 & 42 & -5 & 36 \\
\hline 7 & 7 & 33 & -73 & 48 & 7 & 28 & -70 & 49 \\
\hline 9 & 5 & 48 & 13 & 37 & 4 & 46 & 14 & 33 \\
\hline 18 & 7 & 19 & -99 & -12 & 4 & 11 & -93 & -10 \\
\hline 19 & 5 & 37 & -91 & -13 & 5 & 19 & -85 & -3 \\
\hline 37 & 8 & 42 & -72 & -18 & 7 & 34 & -74 & -18 \\
\hline 39 & 1 & 25 & -73 & 37 & 2 & 39 & -32 & 14 \\
\hline 40 & 5 & 44 & -58 & 44 & 3 & 34 & -72 & 43 \\
\hline 47 & 3 & 42 & 18 & -2 & 1 & 51 & 23 & -5 \\
\hline insular & & & & & 3 & -32 & 11 & 7 \\
\hline
\end{tabular}

hemisphere (BA 19/37), are involved in both orientation and motion discrimination. These regions have also been implicated in other perceptual tasks, such as object priming (Buckner et al. 1998) and shape analysis (Kanwisher et al. 1997). The PET studies of discrimination have further shown the task-dependent activation in prefrontal areas suggested by the present study: BA 6,9,32 (Dupont et al. 1998; Orban et al. 1998) and right insular cortex (Dupont et al. 1998). Parietal activations have been shown in fMRI experiments on direction discrimination (Cornette et al. 1998), object priming (Buckner et al. 1998), and ERP experiments on delayed spatial frequency discrimination (Reinvang et al. 1998). None of these studies have reported task-dependent activity in regions of the temporal cortex. In the present study, our acquisition volume excluded the anterior temporal pole, so we cannot make any statement on its role in stimulus discrimination.

In the PET experiments on discrimination (Orban et al. 1997, 1998; Cornette et al. 1998; Dupont et al. 1998), discrimination-specific activation of the middle fusiform gyrus of the extrastriate cortex was restricted to the right hemisphere, whereas the fMRI experiment (Cornette et al. 1998) found comparable left-hemisphere activation in four out of five subjects. The present results give no indication of hemispheric asymmetry in spatial frequency discrimination, but this might be a consequence of the particular spatial frequencies of the stimuli. Psychophysical and recent ERP experiments have shown that hemispheric asymmetry in spatial frequency discrimination depends both on the spatial frequency content of the stimuli and on the timing of the under lying process (Christman 1997; Watten et al. 1998; Reinvang, Magnussen, and Greenlee, submitted).

In general, the activation differences were observed in terms of regional differences in cortical activation rather than in the recruitment of specific regions in the discrimination task. The specific role, if any, of the various brain regions in the perceptual-discrimination process remains uncertain. It is likely that many cortical regions are activated by visual-perceptual tasks, and differences between the processing of these tasks reside in the computational algorithms used rather than the number and location of cortical regions involved.

It is known that prefrontal regions are important in encoding and retrieval from episodic memory (Cabeza and Nyberg 1997) and that several prefrontal areas are involved in working memory (Courtney et al. 1997; Petit et al. 1998) and dual tasks (D'Esposito et al. 1995). Empirical evidence suggests that these memory processes are not specifically involved in successive perceptual discrimination with short interstimulus intervals (Dupont et al. 1998; Magnussen et al. 1998; Magnussen and Greenlee 1999). Extracting the spatial frequency difference between two vertical gratings presented among horizontal distractors could be thought of as a workingmemory task. It is, thus, likely that any perceptual task drawing on the processing capacity of focal attention is likely to invoke automatically episodic memory, even if the particular experimental task does not require memory retrieval (as was the case for our control task). In light of our earlier ERP work (Reinvang et al. 1998) and the present results, we would like to suggest that regions of 
parietal cortex (BA 7/40) are the best candidates for the location of the discrimination component in successive discrimination of elementary visual features.

Acknowledgements This study was supported by grants from the Norwegian Research Council MH (SM and IR), the Alexander von Humboldt Foundation (SM), the Hermann-und-Lilly-Schilling Stiftung, and the Deutsche Forschungsgemeinschaft (Gr988/15). The authors thank Jürgen Hennig for providing access to the imaging facilities.

\section{References}

Buckner RL, Goodman J, Burock M, Rotte M, Koutstaal W, Schacter D, Rosen B, Dale AM (1998) Functional-anatomic correlates of object priming in humans revealed by rapid presentation event-related fMRI. Neuron 20:285-296

Cabeza R, Nyberg L (1997) Imaging cognition: an empirical review of PET studies with normal subjects. J Cogn Neurosci $9: 1-26$

Christman S (1997) Hemispheric asymmetry in the processing of spatial frequency: experiments using gratings and bandpass filtering. In: Christman S (ed) Cerebral asymmetries in sensory and perceptual processing. Elsevier, Amsterdam, pp 3-30

Cornette L, Dupont P, Rosier A, Sunart S, Van Hecke P, Michiels J, Mortelmans L, Orban GA (1998) Human brain regions involved in direction discrimination. J Neurophysiol 79:2749_ 2765

Courtney SM, Ungerleider LG, Keil K, Haxby JV (1997) Transient and sustained activity in a distributed neural system for human working memory. Nature 386:608-611

Cox RW (1996) AFNI: Software for analysis and visualization of functional magnetic neuroimages. Comput Biomed Res 29: 162-173

D'Esposito M, Detre JA, Alsop DC, Shin RK, Atlas S, Grossman M (1995) The neural basis of the central executive system of working memory. Nature 378:279-81
Dupont P, Vogels R, Vandenberghe R, Rosier A, Cornette L, Bormans G, Mortelmans L, Orban GA (1998) Regions in the human brain activated by simultaneous orientation discrimination: a study with positron emission tomography. Eur J Neurosci 10:3689-3699

Kanwisher N, Woods RP, Iacoboni M, Mazziotta JC (1997) A locus in human extrastriate cortex for visual shape analysis. J Cogn Neurosci 9:133-142

Magnussen S, Greenlee MW (1999) The psychophysics of perceptual memory. Psychol Res 62:81-92

Magnussen S, Idås E, Holst Myhre S (1998) Representation of orientation and spatial frequency in perception and memory. A choice reaction time analysis J Exp Psychol Hum Percept Perform 24:707-718

Orban GA, Vogels R (1998) The neuronal machinery involved in successive orientation discrimination. Prog Neurobiol 55:117147

Orban GA, Dupont P, Vogels R, Bormans G, Mortelmans L (1997) Human brain activity related to orientation discrimination. Eur J Neurosci 9:246-259

Orban GA, Dupont P, De Bruyn B, Vandenberghe R, Rosier A, Mortelmans L (1998) Human brain activity related to speed discrimination tasks. Exp Brain Res 122:9-22

Petit L, Courtney SM, Ungerleider LG, Haxby JV (1998) Sustained activity in the medial wall during working memory delays. J Neurosci 18:9429-9437

Reinvang I, Magnussen S, Greenlee MW, Larsson PG (1998) Electrophysiological localization of brain regions involved in perceptual memory. Exp Brain Res 123:481-484

Scherg M (1996) BESA Version 2.2. Megis Software $\mathrm{GmbH}$, Munich

Smith AT, Greenlee MW, Singh KD, Kraemer FM, Hennig J (1998) The processing of first- and second-order motion in human visual cortex assessed by functional magnetic resonance imaging (fMRI). J Neurosci 18:3816-3830

Talairach J, Tournoux P (1988) Co-planar stereotaxic atlas of the human brain. Thieme, New York

Watten R, Magnussen S, Greenlee MW (1998) Spatial frequency discrimination, brain lateralization and acute intake of alcohol Perception 27:729-736 